\title{
Modelling and Robust Control of Twin Rotor MIMO System
}

\author{
Syed Humayoon Shah \\ Dept. of Mechatronics Engineering, \\ University of Engineering and Technology, \\ Peshawar, Pakistan \\ engrhumayoon@yahoo.com
}

\author{
Said G Khan \\ Dept. of Mechanical Engineering, \\ CoE Yanbu, Taibah University, \\ Al-Madinah, Saudi Arabia \\ sfatehrahman@taibahu.edu.sa
}

\author{
Jamshed Iqbal \\ Dept. of Electrical and \\ Electronic Engineering, \\ University of Jeddah \\ Jeddah, Saudi Arabia \\ jmiqbal@uj.edu.sa
}

\author{
Mathkar Alharthi \\ Dept. of Chemical Engineering, \\ CoE Yanbu, Taibah University, \\ Al-Madinah, Saudi Arabia \\ maaharthi@taibahu.edu.sa
}

\begin{abstract}
Recently, unmanned aerial vehicles (UAVs) have witnessed immense popularity in various fields, ranging from surveillance, rescue, and fire fighting to other more sophisticated military and commercial applications. However, due to their highly nonlinear nature and dynamic operational environment, the control of UAVs is still a challenging task. Linear QuadraticGaussian Regulator (LQG), is an optimal control technique, which has been very popular for UAVs control. However, for robust performance, an accurate dynamic model of a system is required. In order, to overcome this limitation, the present work couples an integral sliding mode controller with the LQG controller to deal with the modelling inaccuracies. Experimental results of pitch control of the laboratory-based twin rotor MIMO system (TRMS), validate the performance of ISMC-LQG controller.
\end{abstract}

Index Terms-Twin rotor multiple input multiple output system, LQG, Kalman filter, ISMC

\section{INTRODUCTION}

Twin rotor multiple input multiple output system (TRMS) is a highly nonlinear system developed by Feedback Instruments Limited as shown in Figure 1. It is an aerodynamic system similar to a helicopter having two Degrees of Freedom (DOF). The associated experimental setup model mainly consists of a beam having rotors on each side. A propeller attached to each rotor produces thrust, which is responsible for vertical (pitch) and horizontal (yaw) motion. The entire system is highly complex due to nonlinearity and cross-coupling.

A plethora of literature is available on control of TRMS [1], [2]. Wen and Li designed an optimal control based on Proportional Integral Derivative (PID) and deadbeat approach and used for control of decoupled SISO TRMS [3]. Biswas et al. employed particle swarm optimization based PID control technique to keep the tracking error minimum by automatically tuning gains of the PID controller [4]. Another research [5] presented control of TRMS with the help of derivative filter coefficient coupled with PID controller and then compared with conventional PID controller. In addition, for control of yaw and pitch angles of the twin rotor, fuzzy sliding mode control techniques have been used [6]. Moreover, to cover various operating areas fuzzy Linear Quadratic Regulator (LQG) is employed for control of pitch and yaw angles [7], [8]. It is pertinent to mention here that, model uncertainties and disturbances highly affect the performance of controller. To eliminate model uncertainties and disturbances of the TRMS, a feedback linearization control approach is used by Karimi and Motlag [9]. An accurate model is needed to cancel out nonlinearities which are often very difficult to estimate. Thus, to overcome model non-linearities, a model reference adaptive control technique is employed to make the system move quickly and precisely to the desired requirements dictated by a reference model [10].

Control of such a non-linear systems is a highly challenging task [11]. Linear Quadratic Gaussian (LQG) controller is an optimal and off-line control technique which is extensively used to control a TRMS [12], [13]. However, LQG requires an accurate model of the system which is somehow not possible in case of nonlinear systems [14]. Integral Sliding Mode Control (ISMC) [15] is a robust control technique which is coupled with LQG in this work to overcome the issue of deriving exact model of a TRMS.

This paper is organized as follows: Section II derives and analyzes model of a TRMS. Section III presents the experi- 


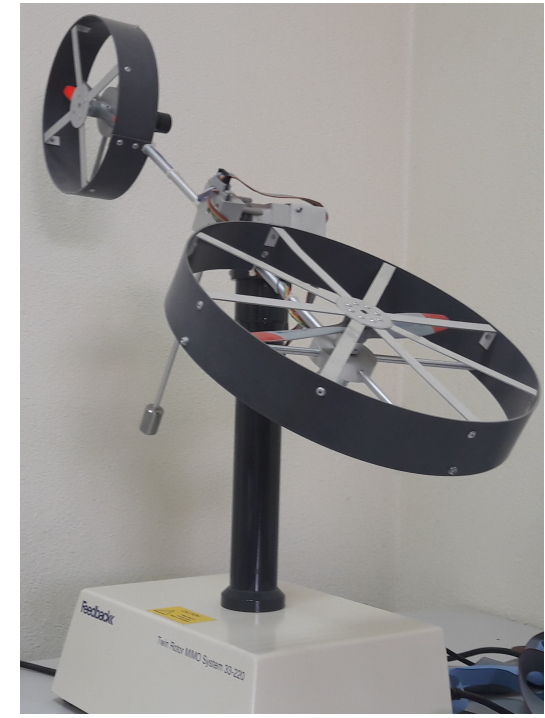

Fig. 1. Twin Rotor Multiple Input Multiple Output system

mental results. Finally, section IV discusses the conclusion.

\section{LQG CONTROL OF TRMS}

The optimal control with $L Q R$ has a major drawback i.e. the need of full state feedback which may not always be possible because of cost factor or unavailability of sensors or due to any other reasons [16]. To overcome this, Kalman filter is used to estimate the unavailable states and to fulfill the need of full state feedback for an LQR. The combination of these two makes an LQG controller (see Fig. 2). LQG got its name for the fact that it is applied to linear systems and its cost function is quadratic while the process is assumed to be contaminated with Gaussian noise.

LQG is a modern optimal control methodology and is capable of achieving high control objectives and has high gain and phase margins. LQR produces control gains while Kalman filter estimates the unknown states and filters the noise. The combination of $\mathrm{LQR}$ and Kalman filter is known as the "separation principle". LQG has four design matrices to be optimized; $\mathrm{Q}$ is the state weighting matrix, $\mathrm{R}$ is controlled weighting matrix, $\mathrm{W}$ is a process noise covariance matrix and $\mathrm{V}$ is measurement noise covariance matrix.

\section{A. Modelling}

In order to design the LQG-based control law and to determine the required weights for the Kalman filter, a discrete state-space model of the system needs to be derived. Obtaining a model of the system is extremely important as it allows for conducting close to realistic simulations and comes in handy when optimal control is required. In our model, the inputs, states, and outputs of the system at discrete time step $k$ are denoted by $\mathbf{u}[k], \mathbf{x}[k]$ and $\mathbf{y}[k]$, respectively.

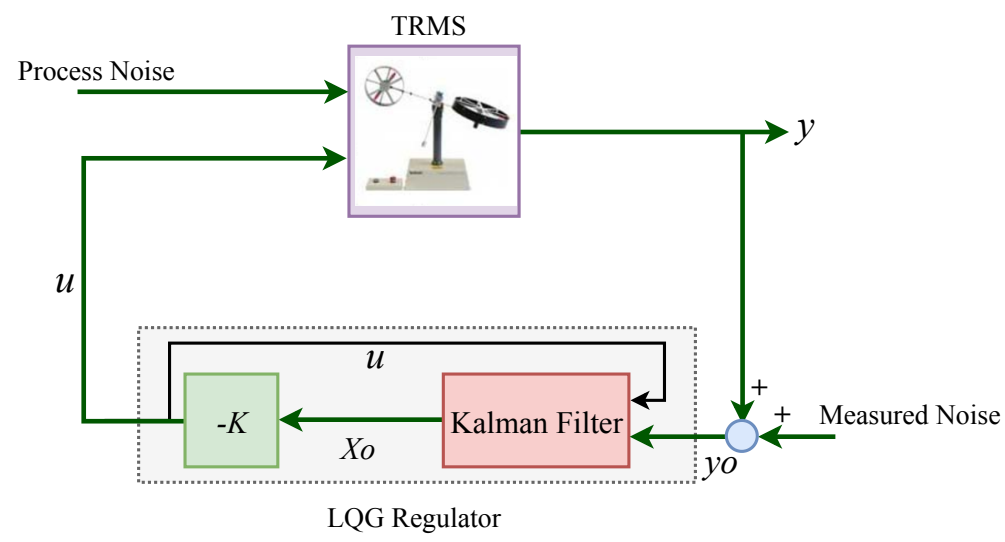

Fig. 2. LQG

The state-space model can be described by

$$
\left\{\begin{array}{l}
\mathbf{x}[k+1]=\mathbf{A x}[k]+\mathbf{B} u[k]+\mathbf{w}[k] \\
y[k]=\mathbf{C x}[k]+D u[k]+v[k]
\end{array}\right.
$$

where matrix $\mathbf{A}, \mathbf{B}, \mathbf{C}$, and $\mathbf{D}$ represent system state matrix, input matrix, output matrix and coupling matrix. The vectors $\mathbf{w}[k]$ and $v[k]$ show the white Gaussian process and measurement noise with the corresponding covariance matrices respectively represented by $\mathbf{W}$ and $\mathbf{V}$.

In order to obtain a concrete model, the system was supplied with a pseudo-random binary sequence (PRBS) and multi-step inputs. The responses were recorded over a window of 100 seconds. The input voltage and pitch output of the system were recorded and imported into the MATLAB's ident system identification toolbox to derive the closest possible model. Consequently, state-space model of the TRMS four matrices $(\mathrm{A}, \mathrm{B}, \mathrm{C}$, and $\mathrm{D})$ in (1) were estimated to be

$$
\begin{aligned}
& \mathbf{A}=\left[\begin{array}{cccc}
0.9551 & -0.1940 & 0.0149 & -0.0079 \\
0.1783 & 0.9698 & 0.1047 & 0.0275 \\
0.0381 & -0.0755 & 0.9406 & -0.2445 \\
0.0195 & 0.0124 & -0.0238 & -0.8359
\end{array}\right] \\
& \mathbf{B}=\left[\begin{array}{l}
-0.0119 \\
-0.0171 \\
-0.0621 \\
-0.1602
\end{array}\right] \\
& \mathbf{C}=\left[\begin{array}{llll}
0.9754 & -0.0800 & -0.0407 & 0.0001
\end{array}\right]
\end{aligned}
$$

and $D=0$, which means that no direct feedthrough exists between the input and output of the TRMS. Series of experiments on the hardware platform lead the selection of above mentioned fourth-order model owing to its close matching with the behavior of the real system. Lower ordered models did not match very well, which may be attributed to the high nonlinear behaviour of the system. 


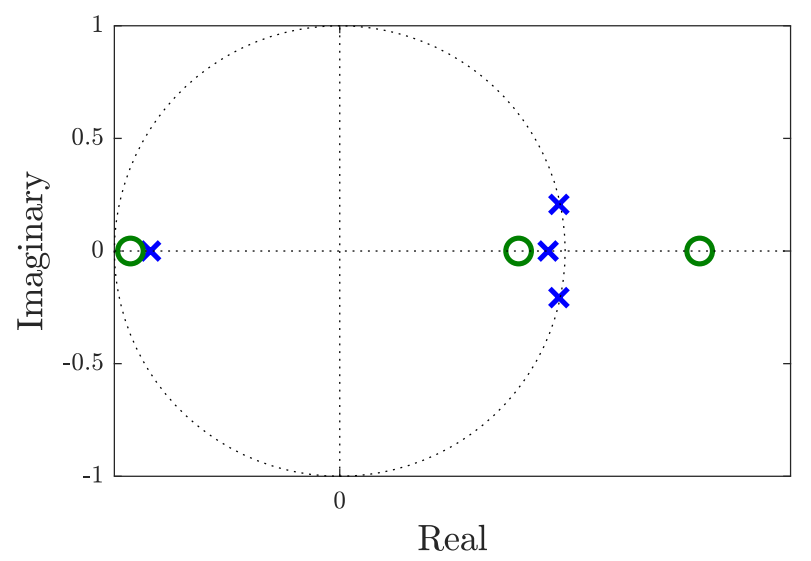

Fig. 3. Pole $(\mathrm{X})$-zero $(\mathrm{O})$ plot of the derived system model. The Unit circle is also shown

\section{B. Model Analysis}

The derived model was examined from different perspectives. As shown in Figure 3, there are four poles of the identified model consisting of a pair of complex poles representing the oscillatory nature of the TRMS, which makes it particularly difficult to control. The two poles are very close to the edge of the unit circle, thereby making the model marginally stable. There is also one zero outside the unit circle implying a nonminimum-phase system. A non-minimum phase system brings more constraints such as limited control bandwidth [17].

Figure 4 shows the transient response of the derived model when the system is excited with a unit step. The settling time can be seen to be around 52 seconds, which apparently seems reasonably long. However, this value is confirmed from the experimental setup which resulted in approximately the same settling time.

Figure 5 shows the autocorrelation of the output pitch residuals and the cross-correlation between the input and output residuals. As illustrated with the dashed lines, both of

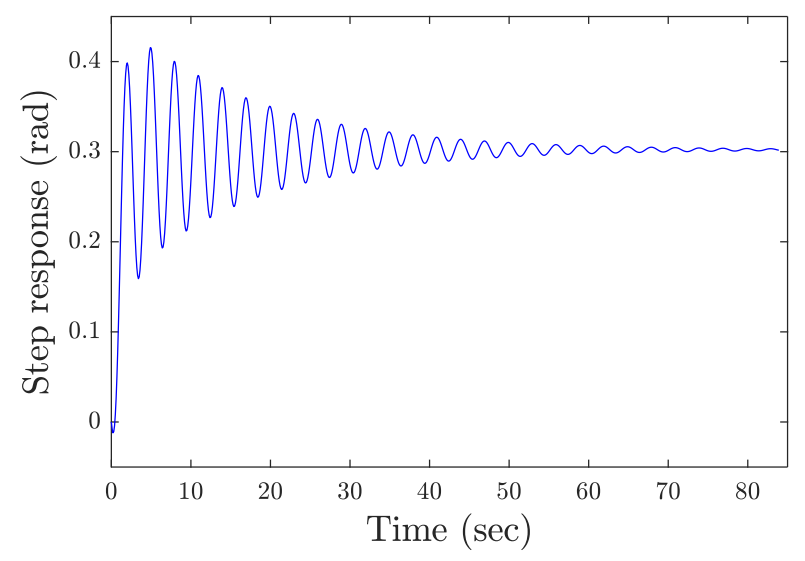

Fig. 4. Transient response of the derived system model when subjected to a unit step
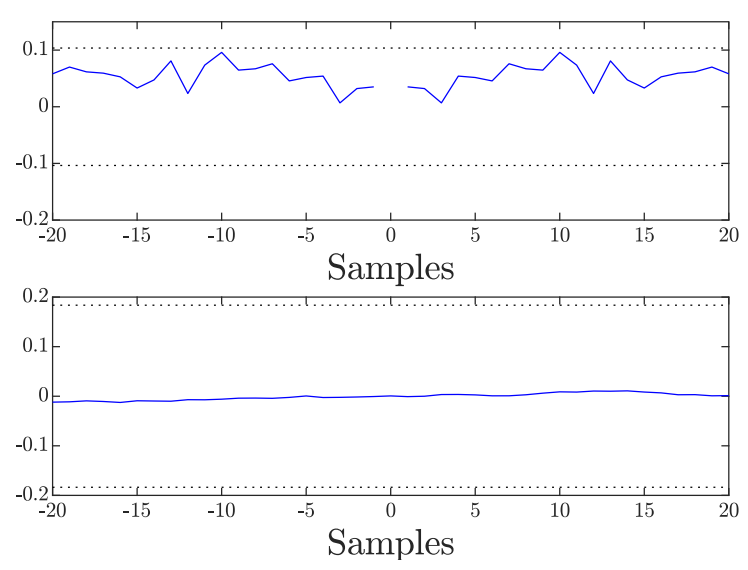

Fig. 5. The autocorrelation of the output residuals (top) and the crosscorrelation of the input and output residuals (bottom)

the correlation sequences lie within the acceptable confidence limits. These results confirm the stability of the model and suggest that it serves as a good fit for the actual system. The similarity percentage was approximately over $81 \%$. Figure 6 shows the measured pitch output of the system compared to that obtained by the model using the mean square error approach. Close matching of the two waveforms is evident. The little difference observed is due to model imperfections as the system is highly nonlinear in nature. MATLAB/Simulink identification toolbox was employed for plotting and comparing simulated output and measured output.

\section{EXPERIMENTAL RESULTS}

The LQG controller was experimentally tested on the real TRMS. The pitch angle reference position was set to a square wave (see Figure 7). The LQR and the Kalman filters are tuned to get better tracking performance with minimum oscillation levels. However, even with many trials and using different values of $\mathrm{Q}, \mathrm{R}, \mathrm{W}$ and $\mathrm{V}$, matrices, better results could not be obtained with an LQG. Therefore, LQG was enhanced with

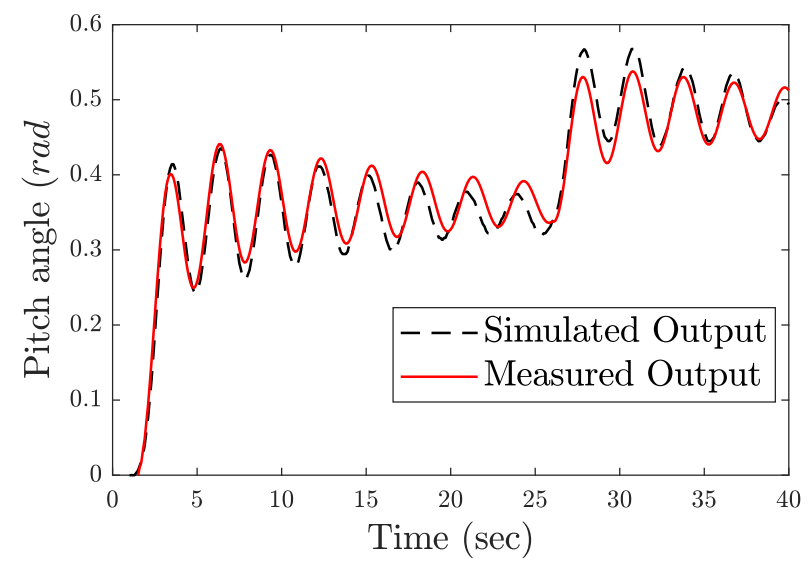

Fig. 6. Measured and simulated outputs 
an integral sliding mode element [15] and tested. The results were improved significantly as demonstrated in Figure 8.
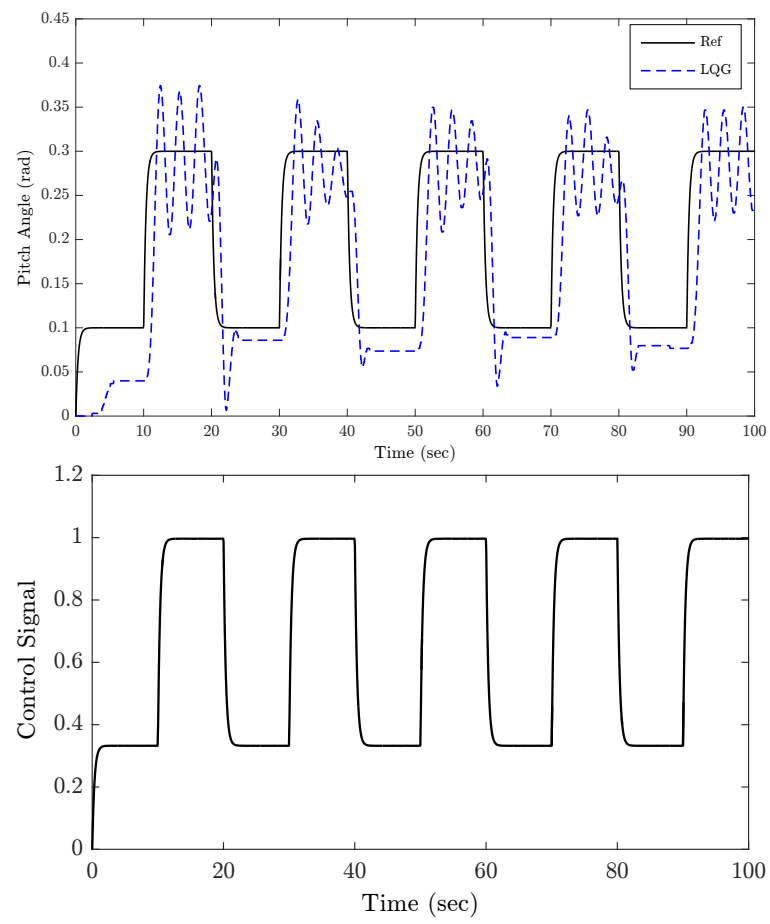

Fig. 7. Experimental Result: the input and output of the TRMS system (top) and control signal (bottom) using the LQG controller
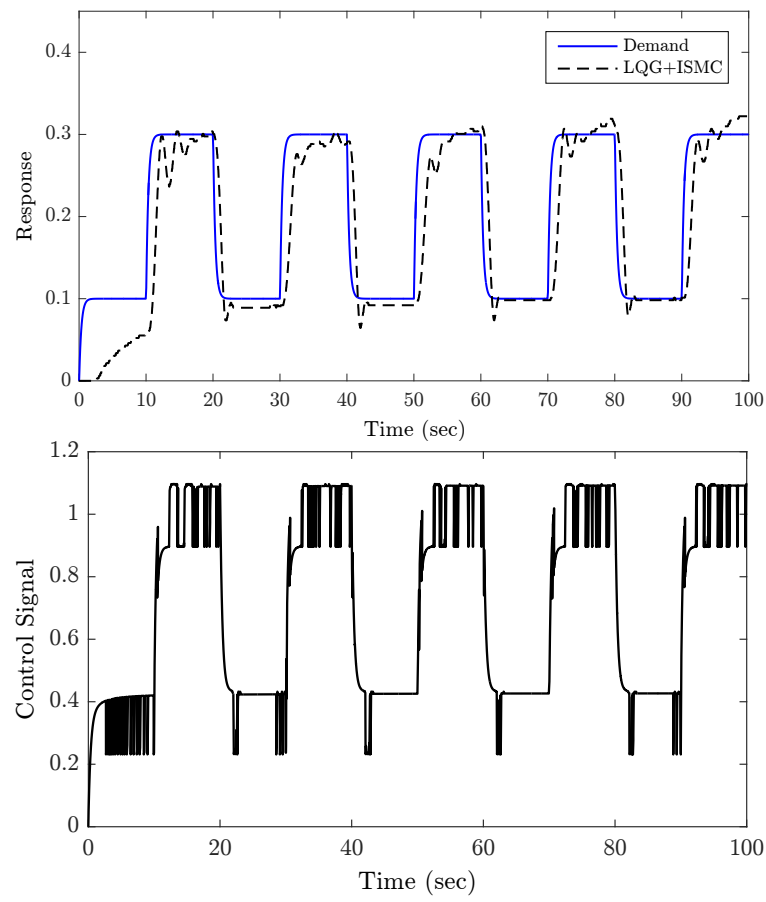

Fig. 8. ISMC-LQG experimental results for a square wave.

\section{CONCLUSION}

TRMS is a highly sophisticated experimental setup commonly used in the academic environment for controller testing. Control of such a system is very complex due to its inherent nonlinear dynamics. In this work, LQR and Kalman filter are coupled (LQG) to devise an optimal control scheme for the pitch. Precise model of the system is required to realize better tracking performance in case of employing the LQG controller, which is often not easy. Thus, for tackling this issue, an ISMC controller is coupled with the LQG in this paper. The experimental results obtained validate the performance of the MIMO control scheme.

\section{REFERENCES}

[1] S. Miah, M. R. Kafi, and H. Chaoui, "Generalized cascaded control technology for a twin-rotor mimo system with state estimation," Journal of Control, Automation and Electrical Systems, vol. 30, no. 2, pp. 170180, 2019.

[2] W. Netto, R. Lakhani, and M. S. Sundaram, "Design and performance comparison of different adaptive control schemes for pitch angle control in a twin-rotor-mimo-system," International Journal of Electrical and Computer Engineering, vol. 9, no. 5, pp. 4114-4129, 2019.

[3] P. Wen and Y. Li, "Twin rotor system modeling, de-coupling and optimal control," in International Conference on Mechatronics and Automation. IEEE, 2011, pp. 1839-1842.

[4] P. Biswas, R. Maiti, A. Kolay, K. D. Sharma, and G. Sarkar, "Pso based pid controller design for twin rotor mimo system," in International Conference on Control, Instrumentation, Energy and Communication. IEEE, 2014, pp. 56-60.

[5] S. K. Pandey and V. Laxmi, "Control of twin rotor mimo system using pid controller with derivative filter coefficient," in Students' Conference on Electrical, Electronics and Computer Science. IEEE, 2014, pp. 1-6.

[6] C.-W. Tao, J.-S. Taur, Y.-H. Chang, and C.-W. Chang, "A novel fuzzy-sliding and fuzzy-integral-sliding controller for the twin-rotor multi-input-multi-output system," IEEE Transactions on Fuzzy Systems, vol. 18, no. 5, pp. 893-905, 2010.

[7] C.-W. Tao, J.-S. Taur, and Y. Chen, "Design of a parallel distributed fuzzy lqr controller for the twin rotor multi-input multi-output system," Fuzzy Sets and Systems, vol. 161, no. 15, pp. 2081-2103, 2010.

[8] S. G. Khan, W. Naeem, R. Sutton, and S. Sharma, "Application of soft computing techniques to a lqg controller design," in Advanced Design and Manufacture to Gain a Competitive Edge, X.-T. Yan, C. Jiang, and B. Eynard, Eds. London: Springer London, 2008, pp. 137-146.

[9] H. R. Karimi and M. R. J. Motlagh, "Robust feedback linearization control for a non linearizable mimo nonlinear system in the presence of model uncertainties," in International Conference on Service Operations and Logistics, and Informatics. IEEE, 2006, pp. 965-970.

[10] A. Chelihi and M. Chemachema, "Model reference adaptive control for twin rotor multiple-input and multiple-output system via minimal controller synthesis," Proceedings of the Institution of Mechanical Engineers, Part I: Journal of Systems and Control Engineering, vol. 228, no. 6, pp. 406-418, 2014.

[11] S. A. Ajwad, J. Iqbal, R. U. Islam, A. Alsheikhy, A. Almeshal, and A. Mehmood, "Optimal and robust control of multi dof robotic manipulator: Design and hardware realization," Cybernetics and Systems, vol. 49, no. 1, pp. 77-93, 2018.

[12] V. S. Rao, V. George, S. Kamath, and C. Shreesha, "Comparison of lqg controller with reliable $\mathrm{h}$ infinity controller designed for trms," International Journal of Control Theory and Applications, vol. 8, no. 3, pp. 1171-1179, 2015.

[13] D. Y. Dube and H. G. Patel, "Suppressing the noise in measured signals for the control of helicopters," Fluctuation and Noise Letters, vol. 18, no. 01, p. 1950002, 2019.

[14] J. Iqbal, M. Ullah, S. G. Khan, B. Khelifa, and S. Ćuković, "Nonlinear control systems-a brief overview of historical and recent advances," Nonlinear Engineering, vol. 6, no. 4, pp. 301-312, 2017.

[15] S. H. Shah, S. Khan, I. ul Haq, K. Shah, and A. Abid, "Compliance control of robotic walk assist device via integral sliding mode control," in International Bhurban Conference on Applied Sciences and Technology. IEEE, 2019, pp. 515-520. 
[16] S. Ajwad, M. Ullah, B. Khelifa, and J. Iqbal, "A comprehensive stateof-the-art on control of industrial articulated robots," Journal of Balkan Tribological Association, vol. 20, no. 4, pp. 499-521, 2014.

[17] I. horowitz, "Invited paper survey of quantitative feedback theory (qft)," International Journal of Control, vol. 53, no. 2, pp. 255-291, 1991. 